\title{
Aula invertida universitaria: evaluación y análisis de los resultados tras su aplicación \\ University flipped classroom: evaluation and analysis of the results after its implementation
}

\author{
Félix Yllana-Prieto, Jin Su Jeong, David González-Gómez \\ feyllanap@unex.es, jin@unex.es, dggomez@unex.es \\ Departamento de Ciencias Experimentales y Matemáticas \\ Universidad de Extremadura \\ Cáceres, España
}

\begin{abstract}
Resumen- El aula invertida o "flipped classroom" es una metodología de aprendizaje en la que la enseñanza tradicional se "invierte". Las explicaciones del profesor se proporcionan mediante video-lecciones y actividades, entre otros recursos, que los estudiantes ven de forma individualizada o grupal antes de clase. De esta forma, en el aula se pueden llevar a cabo más actividades de aprendizaje centradas en el alumno, permitiendo que las asignaturas tengan un carácter más interactivo. Esta metodología de instrucción sostiene que resulta beneficiosa en la participación y el compromiso de los estudiantes, logrando un aprendizaje más significativo que con una metodología de instrucción tradicional. Este trabajo estudia los efectos del aula invertida en el rendimiento, la percepción y la emoción para maestros en formación en ciencias durante dos cursos. Los resultados indican que los estudiantes mostraron percepciones favorables y emociones positivas hacia el aprendizaje de contenidos científicos al seguir el modelo de aula invertida.
\end{abstract}

Palabras clave: Aula invertida, enseñanza de las ciencias, rendimiento, percepciones, emociones.

Abstract- Flipped classroom is a learning methodology in which traditional teaching is "inverted". The teacher's explanations are provided through video-lessons that students watch individually before class. Here, more time is available in the classroom for more studentcentred learning activities, allowing courses to become more interactive. This instructional methodology claims to be beneficial in student participation and engagement, achieving more meaningful learning than with a traditional instructional methodology. This research studies the effects of the flipped classroom on performance, perception and emotion for teacher trainees in science over three courses. The results indicate that students showed favourable perceptions and positive emotions towards learning science content when following the flipped classroom model.

Keywords: Flipped classroom, science education, performance, perceptions, emotions.

\section{INTRODUCCIÓN}

El modelo del aula invertida introducido por Bergmann y Sams es una metodología relativamente nueva (Munir et al., 2018) fundamentada en el constructivismo y la teoría del aprendizaje social (González-Gómez et al., 2018). Esta metodología se sustenta en la premisa de que la instrucción directa es más eficaz si se realiza de forma individualizada de forma asincrónica mediante el empleo de material interactivo (Munir et al., 2018). Al sacar la instrucción directa del aula, se puede usar el tiempo de clase para llevar a cabo más actividades de aprendizaje centradas en el alumno, permitiendo que las asignaturas tengan un carácter más interactivo (Mohamed y Lamina, 2018). En particular, los agentes principales de esta metodología son los estudiantes, que tienen mayor responsabilidad en el proceso de aprendizaje.

Aunque la aplicación de la metodología de aula invertida está extendiéndose de forma rápida, es cierto que la información existente sobre los resultados de su implementación en niveles universitarios es aún escasa, especialmente en el ámbito de las STEM (Science, Technology, Engineering and Mathematics). Los estudios disponibles indican que el empleo del aula invertida mejora el rendimiento de los estudiantes de forma significativa en las asignaturas relacionadas con las STEM (Love et al., 2013; Yllana-Prieto et al., 2021a). Además, existe un consenso amplio en relación con la percepción positiva que tienen los estudiantes hacia esta novedosa metodología (González-Gómez et al., 2015). Algunos estudiantes señalan que uno de los grandes beneficios del modelo de aula invertida es la posibilidad de poder revisar de forma reiterativa los materiales empleados en la instrucción directa asincrónica, lo cual permite superar las dificultades y la complejidad de la asignatura (Roach, 2014; Bacuilima-Arévalo et al., 2020).

Según Hargreaves (2000), el aprendizaje no debe entenderse exclusivamente como una transferencia de contenidos del profesor al alumno. En el proceso de enseñanza y aprendizaje, diferentes investigaciones señalan la existencia de una estrecha conexión entre las dimensiones cognitiva y afectiva (Blanco y Blanco, 2021). Estas indican que el rechazo emocional de los estudiantes es una de las principales causas que afectan a las asignaturas suspensas, especialmente en un curso STEM porque muchos estudiantes sienten emociones negativas (Aydogan et al., 2015; Jungert et al., 2020). Consecuentemente, las estrategias y metodologías de enseñanza deben fomentar los estados emocionales positivos, ya que estos favorecen el 
aprendizaje activo en el aula, mientras que estados emocionales negativos lo limitan (Vázquez y Manassero, 2007).

Además, es importante destacar que la mayoría de los estudiantes que acceden a estudios de grado de Educación Primaria, es decir maestros en formación, han cursado bachillerato por el itinerario de Ciencias Sociales o Humanidades, por lo que no tienen ninguna vocación científica, y en muchos casos tienen poca simpatía por estas disciplinas, además de no sentirse capacitados para su enseñanza (González-Gómez et al., 2018; Yllana-Prieto et al., 2021b).

Este estudio pretende analizar el rendimiento, las percepciones y las emociones de maestros en formación matriculados en la asignatura "Didáctica de la Materia y la Energía" impartida en el grado de Educación Primaria cuando se sigue un modelo de aula invertida como metodología de instrucción para contenidos científicos.

\section{CONTEXTO}

En un proceso de enseñanza y aprendizaje, el dominio cognitivo y el dominio afectivo de los estudiantes están interrelacionados y conectados. Esto crea la necesidad de investigar nuevas metodologías que consigan una mayor motivación y compromiso por parte de los estudiantes hacia los contenidos tratados. En este sentido, la metodología de aula invertida es una herramienta útil que puede ser beneficiosa para mejorar las dimensiones afectiva (percepciones y emociones) y cognitiva (rendimiento académico) de los estudiantes. Además, existen pocos estudios en los que se muestren resultados de esta metodología implementada a nivel universitario.

\section{A. Objetivo del estudio}

El objetivo principal de esta investigación es medir y evaluar el rendimiento, las percepciones y las emociones de los estudiantes cuando se sigue un modelo de aula invertida como metodología de instrucción para maestros en formación en educación científica.

\section{B. Muestra y contexto del estudio}

Con el objetivo de evaluar el modelo de aula invertida en relación con el rendimiento, las percepciones y las emociones que presentan los estudiantes, este trabajo se lleva a cabo en la asignatura Didáctica de la Materia y Energía, asignatura de contenido científico impartida en el segundo curso del grado en Educación Primaria en la Universidad de Extremadura.

El estudio se ha llevado a cabo durante dos cursos, 2014/2015 y 2015/2016, y en él han participado un total de 153 estudiantes (65 y 88 estudiantes, respectivamente). El 61\% son hombres y el $39 \%$ mujeres en el curso $2014 / 2015$ y el $65 \%$ son hombres y $35 \%$ mujeres para el curso $2015 / 2016$, siendo la edad promedio de los participantes de 21 años en los dos cursos académicos. La mayoría de los participantes provienen de ciencias sociales (71\% y 63\%, respectivamente) (ver Tabla 1).

Tabla 1. Información sociodemográfica completa de la muestra de estudio.

\begin{tabular}{c|cc} 
Información & $\mathbf{2 0 1 4 / 2 0 1 5}$ & $\mathbf{2 0 1 5} / \mathbf{2 0 1 6}$ \\
\hline Estudiantes & 65 & 88 \\
Hombres & $61 \%$ & $65 \%$ \\
Mujeres & $39 \%$ & $35 \%$
\end{tabular}

\begin{tabular}{|c|cc|} 
Ciencias sociales & $71 \%$ & $63 \%$ \\
Ciencias & $20 \%$ & $18 \%$ \\
Arte & $0 \%$ & $5 \%$ \\
Tecnología & $1 \%$ & $3 \%$ \\
Otros & $8 \%$ & $11 \%$ \\
\hline
\end{tabular}

\section{DESCRIPCIÓN}

\section{A. Instrumentos}

Para la recogida de información en relación con la medida de la percepción y emociones se empleó un cuestionario previamente validado. El cuestionario consta de tres secciones: información sociodemográfica, 8 preguntas sobre la percepción y 8 preguntas sobre emociones.

Con respecto a las preguntas sobre la percepción, se ha aplicado una escala Likert de cinco puntos (5-Totalmente de acuerdo, 1-Totalmente en desacuerdo) donde los estudiantes dan su opinión sobre la intervención realizada.

Con respecto al estudio de las emociones de los participantes, se han considerado dos grupos de emociones: emociones positivas (diversión, confianza, entusiasmo y tranquilidad) y emociones negativas (nerviosismo, preocupación, aburrimiento y miedo). En este caso, los participantes expresan en qué grado han sentido cada emoción mediante una escala de 0 a 10 (donde 0 es no haber la emoción durante la actividad y 10 haberla sentido intensamente).

Con relación al rendimiento académico, simplemente se evaluaron las calificaciones obtenidas para las diferentes actividades llevadas a cabo.

\section{B. Diseño del aula invertida}

El curso se estructuró teniendo en cuenta tanto los contenidos teóricos como prácticos. La asignatura se imparte durante 15 semanas, con un horario de 3 horas semanales dedicadas a trabajar los contenidos teóricos y 1 hora semanal para trabajar los contenidos prácticos (laboratorio).

Los contenidos teóricos se pusieron a disposición de los estudiantes en forma de video-lecciones, que fueron planificadas según el plan docente de la asignatura. Para la disposición de este material se empleó la plataforma virtual "PlayPosit" junto con el campus virtual (Moodle) de la universidad (Figura 1). El tiempo disponible en el aula se empleó para la realización de diferentes actividades de tipo colaborativo, elaboración de proyectos, estudios de caso y para resolver las cuestiones surgidas en el estudio individual llevado a cabo por los estudiantes, lo que se conoce como "just-in-time teaching". 


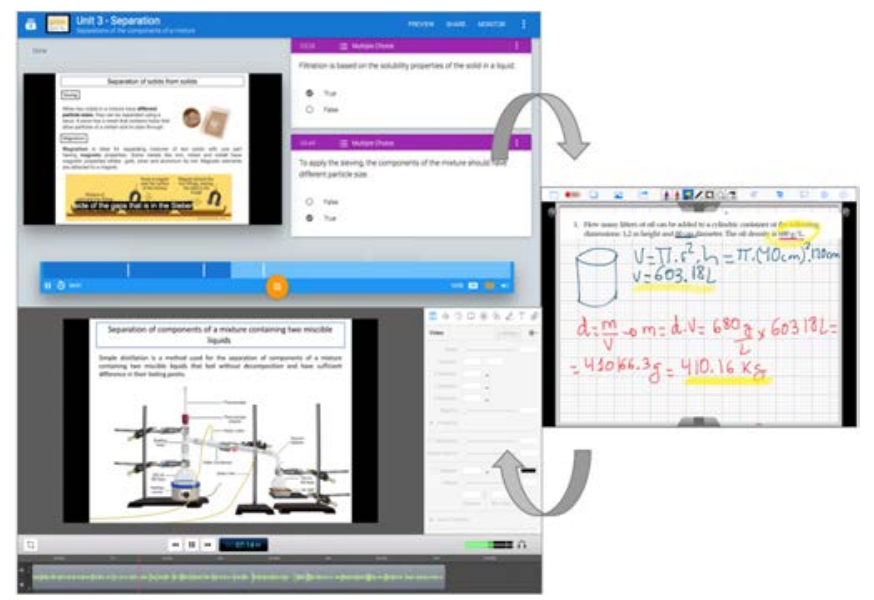

Figura 1. Algunas pantallas de la herramienta "PlayPosit" usada para impartir las video-lecciones e invertir la clase.

\section{Resultados}

\section{A. Análisis y evaluación del rendimiento}

El análisis de los datos estadísticos relativos a la tasa de éxito de esta asignatura, muestran que los estudiantes siempre han tenido algunas dificultades para terminar satisfactoriamente la asignatura propuesta para este estudio. En la Figura 2 se muestra una comparativa de la tasa de resultados del rendimiento académico para los cursos 2014/15 (instrucción tradicional) y 2015/16 (metodología de aula invertida). Ambos grupos tuvieron el mismo tiempo de dedicación a los contenidos.

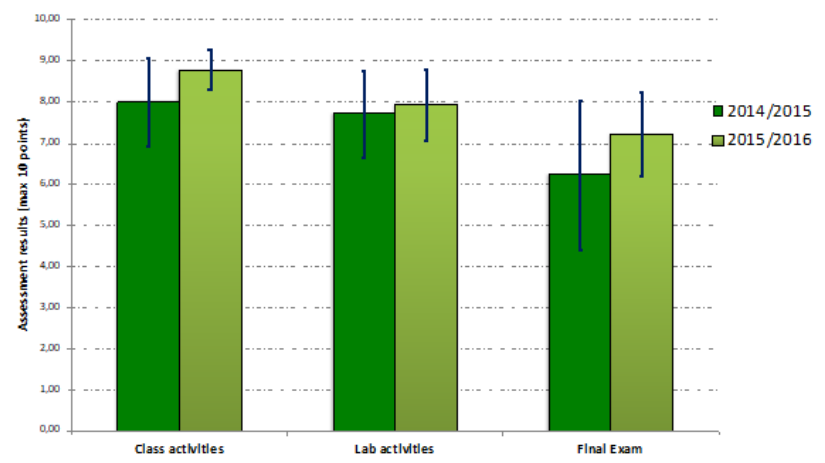

Figura 2. Resultados de las pruebas de evaluación llevadas a cabo durante los cursos 2014/2015 y 2015/2016.

Como se observa en la figura, para el curso en el que se siguió una modalidad de aula invertida, las calificaciones de los estudiantes fueron superiores en comparación al curso anterior, donde se siguió una modalidad tradicional. Este dato es más acentuado para el caso de las calificaciones obtenidas en el examen final, donde los valores medios llegan a duplicarse. En general, para los diferentes tipos de actividades, ejercicios y exámenes en el modelo de aula invertida superaron la tasa de aprobados con respecto a los años anteriores. Estos resultados están en concordancia con los indicados previamente para cursos de ingeniería (Rupakheti et al., 2018), donde también se observaron unos mejores resultados tras la finalización del curso invertido.

\section{B. Análisis y evaluación de la percepción y emociones del alumnado sobre el modelo de aula invertida}

Se han recogido las opiniones de los estudiantes que han recibido los contenidos con la metodología de aula invertida durante el curso 2015/2016. Los resultados obtenidos muestran que hay una percepción general positiva sobre el modelo de aula invertida empleado (Tabla 2). Casi el 97\% de los estudiantes consideran que visualizar las video-lecciones antes de asistir a clase es muy útil para lograr los objetivos de aprendizaje propuestos (A). Por otro lado, a la pregunta sobre si consideran necesarios las video-lecciones para superar los objetivos de la asignatura en las clases teóricas, se observa que tan solo un $14 \%$ de las respuestas no están de acuerdo con esto (B). En este sentido, los estudiantes que no trabajaron las video-lecciones antes de las clases manifestaron que les resultó más complejo participar en las actividades planteadas en el aula. El 95\% de los estudiantes ha visto sus dudas resueltas adecuadamente durante las sesiones de clase (C). Respecto a la percepción y la satisfacción general que ha generado la intervención en las expectativas personales de cada estudiante, todas las respuestas fluctúan entre valores de 5 (totalmente de acuerdo) y 4 (de acuerdo).

Tabla 2. Resultados de las percepciones de los estudiantes tras la intervención (escala Likert de 5 puntos). 5- Totalmente de acuerdo, 4- De acuerdo, 3 Neutro, 2- En desacuerdo, 1Totalmente en desacuerdo.

\begin{tabular}{cccccc} 
& \multicolumn{5}{c}{ Escala Likert } \\
Ítem & $\mathbf{5}$ & $\mathbf{4}$ & $\mathbf{3}$ & $\mathbf{2}$ & $\mathbf{1}$ \\
\cline { 3 - 6 } $\mathbf{A}$ & $54 \%$ & $43 \%$ & $0 \%$ & $0 \%$ & $3 \%$ \\
$\mathbf{B}$ & $28 \%$ & $58 \%$ & $0 \%$ & $11 \%$ & $3 \%$ \\
$\mathbf{C}$ & $30 \%$ & $65 \%$ & $0 \%$ & $5 \%$ & $0 \%$ \\
D & $51 \%$ & $49 \%$ & $0 \%$ & $0 \%$ & $0 \%$
\end{tabular}

En relación con el análisis de la componente afectiva, los estudiantes del curso 2015/2016 expresaron la puntuación más alta en las emociones positivas (diversión, entusiasmo y la confianza). Respecto a las emociones negativas, se observaron puntuaciones más bajas (Figura 3).

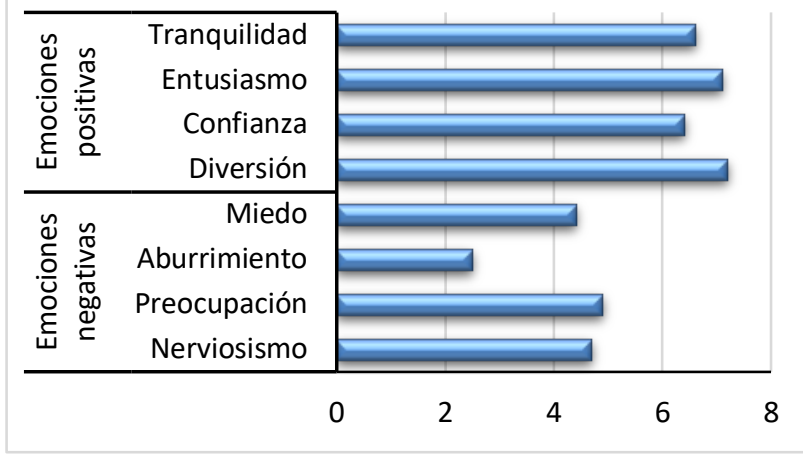

Figura 3. Intensidad de las emociones positivas y negativas mostradas por los estudiantes tras la intervención (escala de 0 a 10).

Respecto al género de los estudiantes, las mujeres expresan valores generalmente más altos de emociones negativas, sin embargo, ambos géneros no tuvieron diferencias en las emociones positivas. Con respecto a la formación preuniversitaria de los estudiantes, los estudiantes que habían 
cursado ciencias y tecnología mostraron valores mayores de confianza y puntuaciones más bajas (por debajo de 4) en las emociones negativas. Los estudiantes de ciencias sociales y arte expresaron puntuaciones más altas en las emociones negativas (más de 5 puntos). Las emociones positivas logran puntuaciones más altas que las negativas, independientemente de la formación académica de los estudiantes.

\section{CONCLUSIONES}

Los resultados muestran que el modelo de aula invertida da mejores resultados que un modelo tradicional expositivo.

En particular, la tasa de aprobados aumentó en más del 10\% en el mismo año para estudiantes por primera vez matriculados. Las calificaciones del examen final fueron notablemente más altas que las calificaciones obtenidas en años anteriores.

En relación con la percepción, los alumnos tienen una opinión general positiva sobre el modelo de aula invertida aplicado. Opinan que tanto el modelo como la lección en vídeo son útiles o muy útiles en el modelo de aula invertida. La posibilidad de ver o volver a ver las lecciones en video ha sido de gran ayuda.

Respecto a las emociones, los estudiantes que asistieron a un modelo de aula invertida han tenido emociones más positivas y menos negativas. Se observa que las puntuaciones más altas se otorgan a las emociones positivas, siendo diversión $\mathrm{y}$ entusiasmo las emociones positivas con valores más altos.

Estos resultados concuerdan con diversos estudios (Abeysekera y Dawson, 2015; Strelan et al., 2020) que observan la mayor eficacia de esta metodología debido al tiempo que pasan los estudiantes realizando actividades prácticas en presencia de un experto que pueda guiarles. En relación con el factor emocional, existen investigaciones recientes que señalan que el modelo de aula invertida proporciona mejora en las emociones positivas que tienen los estudiantes hacia los contenidos impartidos, así como un descenso de las emociones negativas (Jeong et al., 2018; Jdaitawi, 2020).

\section{Agradecimientos}

Los autores agradecen al proyecto de la Consejería de Economía e Infraestructura de la Junta de Extremadura (España) y FEDER (Proyectos IB18004 y GR18004) por hacer posible esta investigación.

\section{REFERENCIAS}

Abeysekera, L., y Dawson, P. (2015). Motivation and cognitive load in the flipped classroom: definition, rationale and a call for research. Higher education research \& development, 34(1), 1-14.

Aydogan, H., Bozkurt, F., y Coskun, H. (2015). An assessment of brain electrical activities of students toward teacher's specific emotions. International Journal of Social, Behaviors, Educational, Economics, Business and Industrial Engineering, 9(6), 1977-2000.

Bacuilima-Arévalo, A. M., García-Herrera, D. G., OchoaEncalada, S. C., y Erazo-Álvarez, J. C. (2020). Google Classroom y Flipped Classroom como estrategias educativas en Educación Básica. EPISTEME KOINONIA, 3(1), 77-96.
Blair, E., Maharaj, C., y Primus, S. (2016). Performance and perception in the flipped classroom. Education and Information Technologies, 21(6), 1465-1482.

Blanco, M. A., y Blanco, M. E. (2021). Bienestar emocional y aprendizaje significativo a través de las TIC en tiempos de pandemia. CIENCIA UNEMI, 14(36), 21-33.

González-Gómez, D., Jeong, J. S., Gallego-Picó, A., y CañadaCañada, F. (2018). Influencia de la metodología flipped en las emociones sentidas por estudiantes del Grado de Educación Primaria en clases de ciencias dependiendo del bachillerato cursado. Educación Química, 29(1), 77-88

González-Gómez, G., Airado Rodríguez, D., Cañada-Cañada, F., y Jeong, J. S. (2015). A Comprehensive application to assist in acid-base titration self-learning: an approach for high school and undergraduate students. Journal of Chemical Education, 92, 855-863.

Hargreaves, A. (2000). Mixed emotions: Teachers' perceptions of their interactions with students. Teaching and Teacher Education, 16(7), 811-826.

Jdaitawi, M. (2020). Does flipped learning promote positive emotions in science education? A comparison between traditional and flipped classroom approaches. Electronic Journal of e-learning, 18(6), 516-524.

Jeong, J. S., y González-Gómez, D. (2018). The study of flipped-classroom for pre-service science teachers. Education Sciences, 8(4), 163.

Jungert, T., Levine, S., y Koestner, R. (2020). Examining how parent and teacher enthusiasm influences motivation and achievement in STEM. The Journal of Educational Research, 113(4), 275-282.

Love, B., Hodge, A., Grandgenett, N., y Swift, A. W. (2013). Student learning and perceptions in a flipped linear algebra course. International Journal of Mathematical Education in Science and Technology, 45(3), 317-324.

Mohamed, H., y Lamina, M. (2018). Implementing flipped classroom that used an intelligent tutoring system into learning process. Computers \& Education, 124, 62-76.

Munir, M. T., Baroutian, S., Young, B. R., y Carter, S. (2018). Flipped classroom with cooperative learning as a cornerstone. Education for Chemical Engineers, 23, 2533.

Roach, T. (2014). Student perceptions toward flipped learning: new methods to increase interaction and active learning in economics. International Review of Economics Education, 17, 74-84.

Rupakheti, C. R., Hays, M., Mohan, S., Chenoweth, S., y Stouder, A. (2018). On a pursuit for perfecting an undergraduate requirements engineering course. Journal of Systems and Software, 144, 366-381.

Strelan, P., Osborn, A., y Palmer, E. (2020). The flipped classroom: A meta-analysis of effects on student performance across disciplines and education levels. Educational Research Review, 30, 100314.

Sun, Z., Xie, K., y Anderman, L. H. (2018). The role of selfregulated learning in students' success in flipped 
undergraduate math courses. The Internet and Higher Education, 36, 41-53.

Vázquez, A., y Manassero, M. A. (2007). En defensa de las actitudes y emociones en la educación científica (I): Evidencias y argumentos generales. Revista Eureka sobre Enseñanza y Divulgación de las Ciencias, 4(2), 247-271.

Yllana-Prieto, F., Jeong, J. S., y González-Gómez, D. (2021a). An Online-Based Edu-Escape Room: A Comparison
Study of Multidimensional Domain of PSTs with a Flipped Sustainability-STEM Contents. Sustainability, 13, 1032 .

Yllana-Prieto, F., Jeong, J. S., y González-Gómez, D. (2021b). Virtual escape room and STEM content: Effects on the affective domain on teacher trainess. JOTSE, 11(2), 331342. 